Trauma Berufskrankh $2006 \cdot 8$ [Suppl 3]:

S264-S268

DOI 10.1007/s10039-006-1154-7

Online publiziert: 12. August 2006

(c) Springer Medizin Verlag 2006

\section{Vorgestellte Studien}

\section{Studie 1}

Der Inhalt der Studie von Hinkeltheim [19] zur Therapie des Schleudertraumas lässt sich wie folgt zusammenfassen:

Das Schleudertrauma der HWS (whiplash) ist eine häufige Verletzung, deren adäquate Therapie für leichte Verletzungsstufen ohne strukturelle Schädigungen fachlich umstritten ist. Dennoch lag es bei einem leichten Schleudertrauma (Grad 1 nach Erdmann), bei dem keine strukturellen Schäden vorlagen, nahe, sich von der Osteopathie - einer funktionellen Methode - Therapieerfolge zu versprechen [24].

Es wurden 100 Patienten untersucht und beobachtet, um die Hypothese zu stützen, dass durch einmalige osteopathische Behandlung eine direkte Verbesserung der HWS-Flexion erreicht wird und dass diese Behandlung eine signifikante Verbesserung der posttraumatischen Bewegungseinschränkung gegenüber der schulmedizinischen Standardtherapie bewirkt.

\section{Studie 2}

In ihr wurde die Rolle der Osteopathie nach Patellarsehnenersatz bei vorderem Kreuzbandriss untersucht [13].

Bei Sportunfällen sind meist die unteren Extremitäten des Körpers betroffen $(72,4 \%)$, wobei mit steigender Tendenz das Kniegelenk mit 12.708 Verletzungen (36,6\%) im Vordergrund steht. Der Autor selbst verfügt in der Arbeit mit Kniepatienten über große Erfahrung (über

P. Fraßmann · Sport- und Rehabilitationszentrum Westend, Berlin

\title{
Osteopathie im Rahmen der Unfall- und Wiederherstellungstherapie
}

3500 Fälle) $[11,12]$ und nahm die im Folgenden beschriebenen Untersuchungen vor.

Für die randomisierte Cross-over-Studie wurden Patienten ausgewählt, die bei einer Operation eine Ersatzplastik des vorderen Kreuzbands mit autologer Patellasehne erhalten hatten. Es wurde eine Zuteilung per Losverfahren in Gruppe A und B vorgenommen. Alle Patienten erhielten eine segmentale Behandlung auf dem Niveau L3 („osteopathic manipulative treatment": OMT) in Kombination mit einem standardisierten Trainingsprogramm, wobei die Behandlungsreihenfolge in den Gruppen variierte. Auch diese Untersuchung sollte auf der Grundlage von Messungen die These belegen, dass die osteopathische Behandlung effektiver wirkt als die schulmedizinische.

\section{Studiendesign und Untersuchungsmethoden}

\section{Studie 1}

Sie wurde über einen Zeitraum von 5 Monaten (01.09.2000-28.02.2001) durchgeführt. Einbezogen wurden Patienten, die mit maximal 6 Wochen altem Schleudertrauma in der Notfallaufnahme der Margarethenklinik Kappeln untersucht bzw. behandelt wurden. Je 50 Teilnehmer wurden als Gruppe A der schulmedizinischen Therapie zugeordnet (Halskrawatte und verordnete 6 Wochen Sportpause), während Gruppe B (ebenfalls 50 Patienten) eine osteopathische Behandlung zuteil wurde, die nach folgendem Schema ablief:

- Phase 1: Mobilisation der Nieren in latero-lateraler Richtung („liegende Acht $^{\prime \prime}=\infty$ )

- Phase 2: Mobilisation der Nieren nach kranial

- Phase 3: Dekoaptationsmanipulation des thorakolumbalen Übergangs (T12 gegenüber L1)

- Phase 4: Mobilisation der Sutura occipitomastoidea

Vor der Behandlung wurde bei jedem $\mathrm{Pa}$ tienten die HWS-Beweglichkeit anhand des Kinn-Jugulum-Abstands gemessen (zunächst in Neutralstellung, dann sowohl in maximaler Extension als auch in maximaler Flexion der HWS). Außerdem wurden die Schmerzen auf der visuellen Analogskala (VAS) bestimmt.

Nach Abschluss der Behandlung wurden nach 15-minütiger Wartezeit erneut die Bewegungsausmaße der HWS (KinnJugulum-Abstand sowie Goniometer) gemessen sowie die Schmerzen nach der VAS überprüft.

\section{Studie 2}

Sie wurde vom 01.07--30.11.2000 im Rehabilitationszentrum Westend in Berlin durchgeführt. Die Patientengruppe, die an
Tab. 1 Schema des Designs von Studie 2

\begin{tabular}{llll}
\hline & 1. Messung & 2. Messung & 3. Messung \\
Gruppe A & Baseline & Nach OMT & Nach Standard \\
\hline Gruppe B & Baseline & Nach Standard & Nach OMT \\
\hline
\end{tabular}




\section{Zusammenfassung $\cdot$ Abstract}

der Studie teilnahm, setzte sich aus sportlich aktiven Personen zusammen (männlich und weiblich, Alter zwischen 18 und 39 Jahren), die sich in der Remodellierungsphase nach einer Operation am vorderen Kreuzband (ACL) befanden. Die Teilnehmer wurden per Losverfahren in 2 Gruppen (A und $B$ ) à 10 Personen eingeteilt. Alle postoperativen ACL-Patienten verfügten zum Zeitpunkt der Studie über ein partielles Bewegungsausmaß im Knie und waren belastungsstabil.

Die Behandlung der Teilnehmer war dualistisch (OMT und MTT) und von 3 Messungen begleitet, die mit dem CYBEX 6ooo durchgeführt wurden. Dieser erfasst:

- maximales Drehmoment [Nm] der Flexoren und Extensoren

- beste Arbeitswiederholung [J] der Flexoren und Extensoren

- Bewegungsausmaß $\left[^{\circ}\right]$

Im Zuge der OMT wurden alle Patienten vom behandelnden Osteopathen auf dem Niveau L3 manipuliert. Bei allen Patienten wurde darüber hinaus eine einheitliche sportphysiotherapeutische Behandlung (MTT) durchgeführt: Dem standardisierten Aufwärmprogramm auf einem Fahrradergometer folgte eine koordinative Trainingsübung auf dem Therapiekreisel (1o Wiederholungen, 3 Serien, 2 min Pause je Serie), der sich nach 3-6 min Ruhezeit eine Übung zur Kräftigung der Beinmuskulatur auf einer „leg press“ anschloss (zu bewegendes Gewicht: 60\% des Eigengewichts des jeweiligen Patienten; 20 Wiederholungen, 4 Serien, 90 s Pause je Serie).

Der Studienverlauf wurde wie in dargestellt $\square$ Tab. 1 gegliedert.

\section{Ergebnisse}

\section{Studie 1}

Um die Verbesserung der HWS-Beweglichkeit durch die jeweils angewandte Therapieform bestimmen zu können, wurde die Differenz der Werte von Neutralstellung, Flexion und Extension, gemessen vor der Behandlung und $15 \mathrm{~min}$ danach, berechnet.

Bei Gruppe A wurde in keinem Fall eine Verbesserung durch die schulmedizi-

Trauma Berufskrankh 2006 · 8[Suppl 3]:S264-S268 DOI 10.1007/s10039-006-1154-7

(c) Springer Medizin Verlag 2006

\section{P. Fraßmann B.Sc. (Hons) Ost.Med. D.O.MROD Osteopathie im Rahmen der Unfall- und Wiederherstellungstherapie}

\section{Zusammenfassung}

Die Osteopathie betont die Beziehung zwischen Struktur und Funktion und legt Wert auf die Selbstheilungskräfte des Köpers. In 2 Studien wurde ihr Wirkungsvermögen im direkten Vergleich zur Schulmedizin untersucht. In der 1. Studie wurde das Schleudertrauma (whiplash) durch Mobilisation der Niere und der Sutura occipitomastoidea behandelt, womit - im Gegensatz zur Standardtherapie (Halskrawatte) - eine höchst signifikante Verbesserung der HWS-Beweglichkeiten und eine Minderung des Schmerzes erzielt werden konnten. In der 2 . Studie wurde die Osteopathie im Rehabilitationsprozess nach Patellarsehnenersatz bei vorderem Kreuzbandriss eingesetzt. Die in 2 Gruppen aufgeteilten Patienten erhielten alle ei-

\section{The significance of osteopathy within the scope of rehabilitation}

\section{Abstract}

A combination of osteopathic treatment and standard medical treatment leads to more effective rehabilitation. To scientifically and quantitatively assess the effectiveness of treatment, patients in two independent studies were divided into groups to receive either osteopathic or standard treatment or to receive a combination of both. Both studies demonstrated the significant efficacy of osteopathy within the scope of rehabilitation. The patients receiving osteopathic therapy experienced immediate improvement of their restrictions, and consequently of their pain. ne Kombination aus osteopathischer (OMT) und sportphysiotherapeutischer Behandlung (MTT), wobei die Behandlungsreihenfolge in den Gruppen getauscht wurde. Nach jeder Teilbehandlung erfolgten Messungen. Die OMT erzielte in allen untersuchten Variablen signifikante Verbesserungen. Beide Studien kamen zu dem Schluss, dass die Behandlungseffekte der Osteopathie umfassender sind als die der standardisierten Therapie.

\section{Schlüsselwörter}

Schleudertrauma · HWS-Beweglichkeit · Vorderer Kreuzbandriss - Osteopathic manipulative treatment (OMT) - Standardisiertes Trainingsprogramm
Treating the entire body as a coherent system, which osteopathy aims to do, appears to lead to the most successful healing. Osteopathy is a therapy that obtains extensive effects. An osteopath is capable of influencing the rehabilitation of both knee and neck injuries (whiplash) in a way that regular medical treatment cannot.

\section{Keywords}

Whiplash - Osteopathic manipulative treatment (OMT) · Significant improvement · Anterior cruciate ligament $(\mathrm{ACL}) \cdot$ Efficacy 


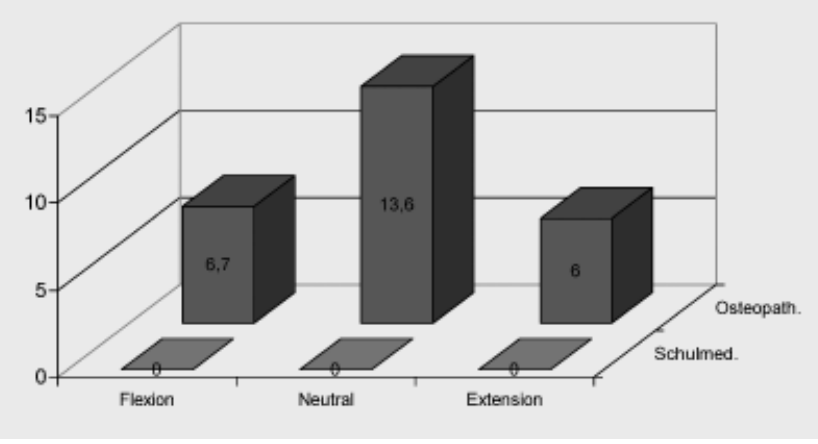

Abb. $1 \Delta$ Verbesserung der HWS nach Therapie, höchstsignifikante Verbesserung von Flexion und Extension direkt nach schulmedizinischer bzw. osteopathischer Therapie sowie höchstsignifikante Verringerung des Kinn-Jugulum-Abstands in Neutralstellung $(p<0,0001)$

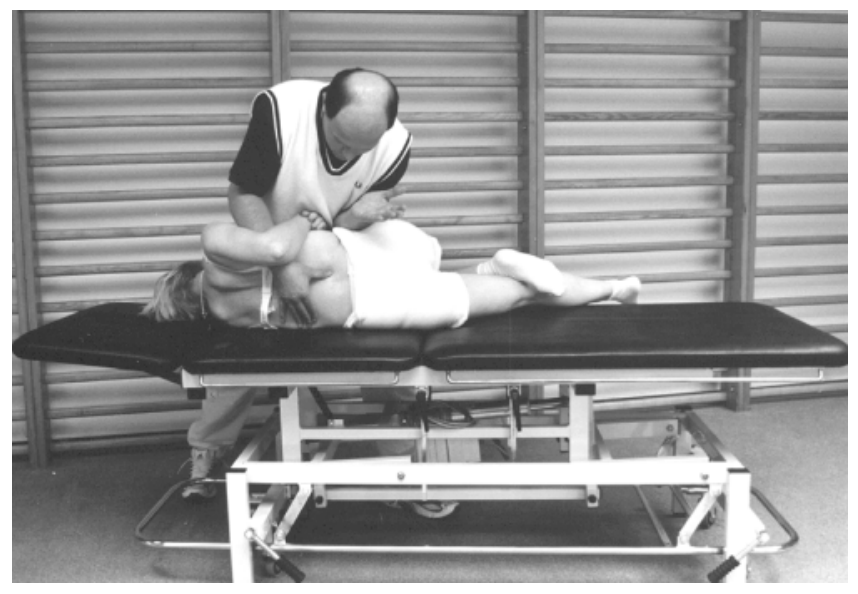

Abb. $3<$ OMT auf Niveau L3

nische Behandlung erzielt. Bei allen Patienten der Gruppe B kam es zu einer Verbesserung der Beweglichkeit. Diese Ergebnisse wurden mit Hilfe des Studentt-Test für verbundene Stichproben statistisch verglichen: Sowohl die Verbesserung von Flexion und Extension als auch die Verringerung des Kinn-Jugulum-Abstands in Neutralstellung waren höchst signifikant ( $\mathrm{p}<\mathrm{o}, \mathrm{ooo1}$ ) (• Abb. 1).

Die Verbesserung des Schmerzes wurde durch Berechnung der Differenz aus den Werten der VAS vor der jeweiligen Therapie und jenen, die $15 \mathrm{~min}$ danach erhoben wurden, bestimmt. Während bei schulmedizinischer Behandlung (Gruppe A) in keinem der 50 Fälle eine Verbesserung zu verzeichnen war, berichteten alle 50 Patienten der Gruppe B nach der osteopathischen Behandlung von einem stark verringerten Schmerzempfinden. Auch diese Ergebnisse wurden nach dem oben genannten Verfahren statistisch verglichen: Die Verbesserung war höchst signifikant (p<0,0oo1) (• Abb. 2).

\section{Studie 2}

Die vom CYBEX 6000 erfassten Daten wurden in 5 abhängige Variablen unterteilt:

$$
\begin{aligned}
& \text { - maximales Drehmoment der Flex- } \\
& \text { oren } \\
& \text { - maximales Drehmoment der Exten- } \\
& \text { soren } \\
& \text { - WBA (beste Arbeitswiederholung) } \\
& \text { der Flexoren } \\
& \text { - WBA (beste Arbeitswiederholung) } \\
& \text { der Extensoren } \\
& \text { - BAM (Bewegungsausmaß) }
\end{aligned}
$$

Die Ergebnisse der 3 Messungen wurden untersucht. Bei Gruppe A kam es nach der erstmaligen Behandlung mit OMT (zwischen der 1. und 2. Messung) zu einer signifikanten Verbesserung in allen abhängigen Variablen, während durch die darauf folgende Standardbehandlung keine signifikanten Verbesserungen zwischen der 2. und 3. Messung mehr zu erzielen waren. Für die Variable „WBA der Extensoren“ ergab sich zu diesem Zeitpunkt so- gar eine signifikante Verschlechterung. Lediglich für BAM kam es zwischen der 2. und 3. Messung zu einer marginalen Verbesserung.

Die Patienten der Gruppe B, die zuerst die Standardtherapie erhielten, wiesen zwischen der 1. und 2. Messung in lediglich 3 abhängigen Variablen signifikante Verbesserungen auf:

- Drehmoment der Flexoren,

- WBA der Flexoren und

- BAM.

Nach der im Anschluss durchgeführten OMT zeigten sich zwischen der 2. und 3. Messung in allen abhängigen Variablen signifikante Verbesserungen (• Tab. 2, 3).

\section{Diskussion}

\section{Studie 1}

Direkt nach der primären Behandlung mit der funktionellen osteopathischen Therapie verbesserte sich die HWS-Beweglichkeit höchstsignifikant in Flexion $(6,7 \mathrm{~mm})$, Extension $(6,0 \mathrm{~mm})$ und in Neutralstellung $(13,6 \mathrm{~mm})$. Nacken- und ventrale Halsmuskulatur wiesen Tonusverringerung bzw. -normalisierung auf. Bei keinem der schulmedizinisch behandelten Patienten zeigte sich eine Veränderung der Werte. Dies war auch prinzipiell zu erwarten, da die Standardtherapie eine Ruhigstellung zur Schonung der HWS zum Ziel hat $[15,28]$.

Die durch die osteopathische Therapie erwirkte Verbesserung erklärt sich durch das biomechanische fasziale und neurologische Prinzip der Bewegungseinschränkung $[5,10]$ : Die Bewegungsschmerzen 
Tab. 2 Overallsignifikanzen der Messwiederholungen

\begin{tabular}{|lll}
\hline Abhängige Variable & Gruppe A & Gruppe B \\
Flexorendrehmoment & 0,0002 & 0,0001 \\
\hline Extensorendrehmoment & 0,0001 & 0,0001 \\
\hline WBA der Flexoren & 0,0001 & 0,0001 \\
\hline WBA der Extensoren & 0,0001 & 0,0001 \\
BAM & 0,0004 & 0,0001
\end{tabular}

Beide Gruppen führen für alle abhängigen Variablen bezüglich ihrer Messwiederholungen zu hochsignifikanten Ergebnissen, $d$. h. beide Gruppen weisen hochsignifikante Unterschiede in den abhängigen Variablen bezüglich zweier oder aller Messzeitpunkte auf. Anhand dieser Ergebnisse kann also nicht auf Unterschiede zwischen den beiden Gruppen infolge unterschiedlicher Behandlungen (Reihenfolge der Behandlung) geschlossen werden

Tab. 3 Signifikanzen der einzelnen Messwiederholungen

\begin{tabular}{lllllll} 
Abhängige Variable & Gruppe A & \multicolumn{5}{c}{ Gruppe B } \\
& $\mathbf{1}$ vs. 2 & $\mathbf{2}$ vs. 3 & $\mathbf{1}$ vs. 3 & $\mathbf{1}$ vs. 2 & $\mathbf{2}$ vs. 3 & $\mathbf{1}$ vs. 3 \\
Flexorendrehmoment & $\mathbf{0 , 0 0 2 1}$ & $\mathbf{0 , 3 4 7 5}$ & $\mathbf{0 , 0 0 1 9}$ & $\mathbf{0 , 0 0 1 7}$ & $\mathbf{0 , 0 0 9 8}$ & $\mathbf{0 , 0 0 0 3}$ \\
\hline Extensorendrehmoment & $\mathbf{0 , 0 0 0 3}$ & $\mathbf{0 , 1 0 5 3}$ & $\mathbf{0 , 0 0 0 5}$ & 0,6557 & 0,0001 & 0,0001 \\
\hline WBA der Flexoren & 0,0014 & 0,3434 & 0,0005 & 0,0015 & 0,0034 & 0,0008 \\
\hline WBA der Extensoren & 0,0025 & 0,0077 & 0,0034 & 0,7791 & 0,0013 & 0,0018 \\
\hline BAM & 0,0143 & 0,0576 & 0,0016 & 0,0022 & 0,0070 & 0,0001
\end{tabular}

Die erstmalige Behandlung mit OMT bei Gruppe A führt signifikante Verbesserungen herbei, die darauf folgende Standardbehandlung vermag dies nicht mehr

Auch bei Gruppe B führte die Standardbehandlung zunächst nur zu einer teilweisen Verbesserung, erst die folgende OMT bewirkte umfassende Verbesserungen

und -störungen der HWS beruhen nicht allein auf den gut untersuchten Bewegungsabläufen der HWS [3, 8], sondern sind v. a. in einer Veränderung der propriorezeptiven Informationen sowie einer Sensibilisierung der zentralen neurologischen Strukturen zu suchen $[6,18]$. Darüber hinaus werden fasziale Retraktionen der Halsfaszien (mit Verbindung zu den Organfaszien) als Ursache für Irritationen der HWS-Region geschildert [9, 20].

Die Messung des Schmerzes erfolgte mit Hilfe der subjektiven VAS, da es sich um eine subjektive Wahrnehmung handelt. Die Qualität der exakten mathematisch-statistischen Berechnung wird dadurch nicht negativ beeinflusst $[1,4]$.

Die Schmerzintensität in der osteopathischen (Mittelwert 4,84) und in der schulmedizinischen Gruppe (Mittelwert 5,36) war etwa gleich. Bei den osteopathisch behandelten Patienten reduzierte sich dieser Wert höchstsignifikant um etwa 2 Punkte auf einen Mittelwert von 2,80, während er bei der schulmedizinischen Kontrollgruppe unverändert blieb.

Somit besteht bei der Therapie des Schleudertraumas mit der in Studie 1 genannten osteopathischen Technik eine wirkungsvolle Behandlungsmethode, die sowohl die Schmerzen als auch die Bewegungsausmaße der HWS signifikant verbessert.

\section{Studie 2}

Bei der differenzierten Betrachtung der Signifikanzen zwischen den jeweiligen in dieser Studie durchgeführten Messungen war nach der erstmaligen Behandlung mit OMT (Gruppe A) eine signifikante Verbesserung in allen abhängigen Variablen auszumachen. Nach der MTT waren keine Verbesserungen festzustellen. In qualitativ hochwertigen Untersuchungen $[16,17]$ wurde der M. quadriceps femoris als Kennmuskel für das Segment $\mathrm{L}_{3}$ bestimmt, d. h. eine somatische Dysfunktion auf dem Niveau $\mathrm{L}_{3}$ bewirkt, dass der M. quadriceps femoris nicht seine volle Kontraktionsfähigkeit besitzt.

Jede vordere Kreuzbandverletzung sowie die anschließende operative Versorgung ziehen $u$. a. ein koordinatives Defizit nach sich $[26,29]$. Als Ursache hierfür werden eine verschlechterte Fähigkeit zur Eigenwahrnehmung (Propriozeption) durch die Verletzung von propriozeptiven Gelenkrezeptoren und ein damit verbundenes vermindertes Koordinationsvermö- gen vermutet [21, 23]. Es wird angenommen, dass das veränderte Feedback der sensorischen Strukturen über die afferente Informationsvermittlung zu Modifikationen auf spinaler und kortikaler Ebene führt. Als efferente Folge einer VKBOperation lassen sich Veränderungen der neuromuskulären Ansteuerungsmuster und des koordinativen Leistungsvermögens feststellen.

Durch eine Manipulation der HVLAthrust-Technik (high velocity - low amplitude) auf das Niveau L3 konnte ein positiver Einfluss auf die Kontraktionsfähigkeit des M. quadriceps femoris durch Parmentier [25] gezeigt werden. Ähnliche Resultate konnten auch in der vorliegenden Studie erzielt werden: Die Komponenten der Flexoren und das BAM hatten sich nach der OMT auf Niveau L3 (• Abb. 3) signifikant verbessert.

Nach dem standardisierten Rehabilitationsprogramm fand bei Gruppe A keine signifikante Verbesserung statt, darüber hinaus ergab sich zwischen der 2. und 3. Messung für die WBA der Extensoren eine signifikante Verschlechterung.

In Gruppe B, in der zuerst das standardisierte Rehabilitationsprogramm zum Einsatz kam, verbesserten sich die Variablen bezüglich der Flexoren und das BAM. Dies ist auf die aktive Stabilisierung durch die ischiokrurale Muskulatur zurückzuführen, die auf neurophysiologischer Ebene mittels Mechanorezeptoren mit dem Kreuzband verbunden ist.

In elektromyographischen Studien nach einer ACL-Operation ließen sich bei unterschiedlichen Testbewegungen veränderte neuromuskuläre Ansteuerungsmuster feststellen $[2,30]$. In der Mehrzahl der Untersuchungen mit dynamischen Testbewegungen konnte ein längerfristig veränderter Synergismus der Muskulatur durch eine geringere Aktivierung des $\mathrm{M}$. vastus medialis und eine verstärkte Aktivierung des M. vastus lateralis und des M. bizeps femoris gemessen werden $[14,27]$.

Durch den Einsatz von OMT zwischen der 2. und 3. Messung zeigten alle abhängigen Variablen signifikante Verbesserungen, die auch bereits bei Gruppe A festgestellt wurden. 


\section{Resümee}

Es muss entschieden werden, welche Form der Nachbehandlung bei einem betroffenen Patienten angewandt werden sollte, um den höchstmöglichen Therapieerfolg zu erzielen. Fest steht, dass es einem postoperativen ACL-Patienten neben einem muskulären Defizit [7] an Bewegungsausmaß [22] und an propriorezeptiven Fähigkeiten [29] fehlt. Die Entscheidung über die Therapieform wird anhand der gesamten Gelenkfunktion getroffen, die sich aus verschiedenen Einzelparametern zusammensetzt (Streckung, Beugung, Schmerzen, Erguss, Muskelzustand, Stabilität, Propriorezeption) und damit als Summationseffekt der Einzelparameter zu definieren ist [29].

\section{Fazit}

Die osteopathische Therapie erreicht - wie in Studie 1 nachgewiesen - im Vergleich zur schulmedizinischen eine höchstsignifikante Verbesserung der Beweglichkeit der HWS. Dadurch sinkt das Schmerzniveau (um durchschnittlich 2 Punkte) unmittelbar, und es werden Folgeerscheinungen aufgrund von eventuellen Haltungsfehlern vermieden. Zusätzlich können durch die Verbesserungen der Schmerzsituation die negativen Folgen eines Schleudertraumas (WAD) gemildert oder sogar verhindert werden, wodurch die Folgekosten gesenkt werden können [28].

Die Bedeutung einer ganzheitlichen Therapie von Patienten mit Schleudertrauma wird an den signifikant besseren Behandlungsergebnissen ersichtlich. Aus den Resultaten der Studie 2 geht eindeutig hervor, dass die OMT-Maßnahmen in allen gemessenen Variablen umfassendere Behandlungseffekte erzielen als das standardisierte Rehabilitationsprogramm. Die Kombination einer osteopathischen Technik mit standardisierten therapeutischen Verfahren induziert eine generelle und grundsätzliche Optimierung der Rehabilitation. Die Rehabilitationsphase wird verkürzt, der Patient schneller arbeitsfähig und alle anfallenden Kosten (Arbeitsausfall, Krankengeld, Behandlungskosten) werden reduziert.
Es gibt keine festen Behandlungspläne für Patienten mit einer vorderen Kreuzbandoperation. Allerdings müssen Grundregeln wie die Berücksichtigung der Zeiträume der Wundheilungsphasen eingehalten werden.

\section{Korrespondierender Autor \\ P. Fraßmann, B.Sc. (Hons) Ost.Med. D.O.MROD}

Sport- und Rehabilitationszentrum Westend, Rüsternallee 14-16, 14050 Berlin peterfrassmann@t-online.de

Interessenkonflikt. Es besteht kein Interessenkonflikt. Der korrespondierende Autor versichert, dass keine Verbindungen mit einer Firma, deren Produkt in dem Artikel genannt ist, oder einer Firma, die ein Konkurrenzprodukt vertreibt, bestehen. Die Präsentation des Themas ist unabhängig und die Darstellung der Inhalte produktneutral.

\section{Literatur}

1. Arendt-Nielsen $L$ (1997) Induction and assessment of experimental pain from human skin, muscle and vicera. In: Jensen TS, Turner JA, WiesenfeldHallin Z (eds) Proceedings of the 8th World Congress on Pain, Progress in Pain Research and Management, vol 8. IASP Press, Seattle, pp 393-425

2. Banzer W, Pfeifer K, Bernhardt M (1994) Neuromuskuläre Aktivierung in der späteren postoperativen Phase bei funktionellen dynamischen Tests. In: Scholle HC, Struppeler A, Freund HJ et al. (Hrsg) Motodiagnostik - Mototherapie. Mayer, Jena, S 253-262

3. Bar HF, Witte HF, Pape HG et al. (1998) Die Bewegungsanalyse der Beschleunigungsverletzung. Orthopädie 27: 827-833

4. Barnsley L, Lord S, Bogduk N (1994) Whiplash injury. Pain 58: 283-307

5. Bogduk N (1986) The anatomy and pathophysiology of whiplash. Clin Biomech 1: 82-101

6. Brault JR, Siegmund GP, Wheeler JB (2000) Cervical muscle response during whiplash: evidence of a lengthening muscle contraction. Clin Biomech 15: 426-435

7. Chen CY, Jiang CC, Jan MH et al. (1995) Role of flexors in knee stability. J Formos Med Assoc 94: 255260

8. Cholewicki J, Panjabi MM, Nibu K et al. (1998) Head kinematics during in vitro whiplash simulation. Accid Anal Prev 30: 469-479

9. Christensen LV, McKay DC (1997) Reflex jaw motions and jaw stiffness pertaining to whiplash injury of the neck. Cranio 15: 242-260

10. Evans RW (1995) Whiplash injuries. In: Evans RW (ed) Neurology and trauma. Saunders, Philadelphia, pp 439-457

11. Fraßmann P (1993) The knee. A handbook for physiotherapists about subchondral lesion, synovial lesion, arthrosis I, II, III. Wissenschaftliche Arbeit, Berlin

12. Fraßmann P (1998) A comparison between two treatments, traction on the knee and compression of the knee. Wissenschaftliche Arbeit, Berlin
13. Fraßmann P (2001) Die Rolle der Osteopathie im Rehabilitationsprozess der 16.-24. Woche nach Patellarsehnenersatz bei vorderem Kreuzbandriss. Ost Med 2001: 20-30

14. Freiwald J, Gnewuch A, Engelhardt M et al. (1998) Trainingstherapie nach Verletzungen des Kniegelenks. Krankengymnastik 2: 228-242

15. Freund BJ, Schwartz M (2000) Treatment of whiplash associated with neck pain with botulinum toxin-A. A pilot study. J Rheumatol 27: 481-484

16. Frisch $\mathrm{H}$ (1995) Neurologische Untersuchungen. In: Frisch H (Hrsg) Programmierte Untersuchung des Bewegungsapparates. Springer, Berlin Heidelberg New York, S 442-513

17. Grieve GP (1982) Segmentale innervatie van huid, spieren en organen. Het mobiliseren van de wervelkolom. De Tijdstroom, Lochem-Poperinge, pp 92-95, 166-170

18. Grifka J, Hedtmann A, Pape HG et al. (1998) Beschleunigungsverletzungen der Halswirbelsäule. Orthopädie 27: 802-812

19. Hinkeltheim A (2001) Fasziale und neurologische Einflüsse von Niere und Sutura occipito-mastoidea auf die Therapie des Schleudertraumas (whiplash). Diplomarbeit, International Academy of Osteopathy, S 18-20

20. Howard RP, Bowles AP, Guzman HM et al. (1998) Head, neck, and mandible dynamics generated by "whiplash". Accid Anal Prev 30: 525-534

21. Irrgang JJ, Whitney SL, Cox ED (1994) Balance and proprioceptive training for rehabilitation of the lower extremity. J Sport Rehabil 1: 68-83

22. Jomah NM, Clingeleffer A, Pinczewski L (2000) Intra-articular mechanical blocs and full extension in patients undergoing anterior cruciate ligament reconstruction. Arthroscopy 16: 156-159

23. Lephart SM, Fu FF (1995) The role of proprioception in the treatment of sports injuries. Sports Exercise Injury 1: 96-102

24. O'Shaughnessy T (1994) Craniomandibular/temporomandibular/cervical implications of a forced hyperextension/ hyperflexion episode (i. e. whiplash). Funct Orthod 11:5-12

25. Parmentier J (2000) The influence of a manipulation of a somatic dysfunction of $L 3$ on the strength of the quadriceps muscle. Durham, USA

26. Pässler HH (1997) Beschleunigte Rehabilitation nach Kreuzbandverletzungen. Arthroskopie 10: 267-273

27. Pfeifer K (1996) Bewegungsverhalten und neuromuskuläre Aktivierung nach Kreuzbandreduktion. Lingua, Neu-Isenburg

28. Schmid P (1999) Whiplash-associated disorders. Schweiz Med Wochenschr 129: 1368-1380

29. Strobel M, Eder K, Eichhorn J (1997) Grundlagen zur Nachbehandlung nach VKB-Rekonstruktion. Arthroskopie 10: 261-266

30. Van Lent ME, Drost MR, Wildenberg FA (1994) EMG profiles of $A C L$-deficient patients during walking: the influence of mild fatigue. Int J Sports Med 15: 508-514 